\section{Making a difference: workforce skills and capacity for integrated care}

\author{
Joy Akehurst \\ Faculty of Health and Wellbeing, University of Sunderland, Sunderland, UK \\ Paul Stronge \\ University of Nottingham, Nottingham, UK, and \\ Karen Giles ${ }^{\circledR}$ and Jonathon Ling \\ Faculty of Health and Wellbeing, University of Sunderland, Sunderland, UK
}

Workforce skills

\begin{abstract}
Purpose - The aim of this action research was to explore, from a workforce and a patient/carer perspective, the skills and the capacity required to deliver integrated care and to inform future workforce development and planning in a new integrated care system in England.

Design/methodology/approach - Semi-structured interviews and focus groups with primary, community, acute care, social care and voluntary care, frontline and managerial staff and with patients and carers receiving these services were undertaken. Data were explored using framework analysis.

Findings - Analysis revealed three overarching themes: achieving teamwork and integration, managing demands on capacity and capability and delivering holistic and user-centred care. An organisational development (OD) process was developed as part of the action research process to facilitate the large-scale workforce changes taking place.
\end{abstract}

Research limitations/implications - This study did not consider workforce development and planning challenges for nursing and care staff in residential, nursing care homes or domiciliary services. This part of the workforce is integral to the care pathways for many patients, and in line with the current emerging national focus on this sector, these groups require further examination. Further, data explore service users' and carers' perspectives on workforce skills. It proved challenging to recruit patient and carer respondents for the research due to the nature of their illnesses.

Practical implications - Many of the required skills already existed within the workforce. The OD process facilitated collaborative learning to enhance skills; however, workforce planning across a whole system has challenges in relation to data gathering and management. Ensuring a focus on workforce development and planning is an important part of integrated care development.

Social implications - This study has implications for social and voluntary sector organisations in respect of inter-agency working practices, as well as the identification of workforce development needs and potential for informing subsequent cross-sector workforce planning arrangements and communication.

Originality/value - This paper helps to identify the issues and benefits of implementing person-centred, integrated teamworking and the implications for workforce planning and OD approaches.

Keywords Integrated care, Workforce development, Workforce planning, Care planning,

Multidisciplinary team

Paper type Research paper

(c) Joy Akehurst, Paul Stronge, Karen Giles and Jonathon Ling. Published by Emerald Publishing Limited. This article is published under the Creative Commons Attribution (CC BY 4.0) licence. Anyone may reproduce, distribute, translate and create derivative works of this article (for both commercial and non-commercial purposes), subject to full attribution to the original publication and authors. The full terms of this licence may be seen at http://creativecommons.org/licences/by/4.0/legalcode

The research team would like to thank all participants who gave their precious time to this research and gave freely their thoughts and ideas to support the development of better services for patients. The authors would also like to thank North East Commissioning Service (NECS) for funding this work.

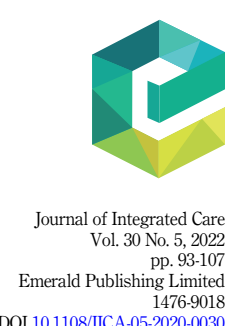

DOI 10.1108/JICA-05-2020-0030 
JICA
30,5

\section{Introduction}

International healthcare strategy is focussed on developing integrated, people-centred care systems, which aim to generate efficiency and quality benefits to the health and care system and the health of the population (WHO, 2020a). Whilst there have been numerous reviews and discussion of evidence in relation to integrated care from a macro- or micro- perspective (WHO, 2020b; Nuffield Trust, 2019; Ling et al., 2012; Goodwin et al., 2014; Schrijvers and Goodwin, 2010), little focus is on the literature about the workforce capacity and capability implications of making the shift to more integrated ways of working. Where there is mention of workforce implications, there tends to be a focus on skill mix, staff substitution, new role development or "service-level integration" rather than whole system integration (Gullery, 2015; Kings Fund, 2020).

In England, the National Health Service (NHS) strategy (NHS, 2019a, b, c) aims to address the challenges of healthcare quality and financial constraints through a range of initiatives including a focus on "workforce" (Dept. Health, 2013; NHS, 2020a, b) and integrated physical and mental healthcare (Das et al., 2016). Alongside the challenges of a changing NHS and the workforce development requirements to address new ways of working, there is a shortage in both the primary care (Health Education England (HEE), 2019) and the nursing workforces (NHS, 2019). There are plans to train more general practitioners (GPs), nurses and midwives and develop new roles to address these gaps, but the transformation in the way staff work together has been acknowledged as a priority (NHS, 2020a, b). The future role and need for career development of the nonprofessional workforce has also been recognised (Health Education, 2018).

Workforce development and planning historically have been in "silos", with organisations planning around their individual requirements rather than an integrated health and social care system. In England, the NHS New Care Models programme (NHS, 2018) was set up to support the development, delivery, evaluation and rapid dissemination of learning from different models of care, known as "vanguards", funded for a fixed term to address, among other issues, the workforce development and planning challenges in integrated care models. Alongside this, the HEE, who are the national body responsible for workforce planning and strategy in the NHS in England, introduced a new single national workforce plan and strategy, though it based on the model of individual professional groups' supply and demand issues.

This research aimed to understand the workforce skills and capacity required in the system to deliver integrated care (Better Sunderland HOMEPAGE, 2019). The vanguard site (one of 26 distributed across England) was a "multispecialty community provider model", and as a change to the existing way these services were configured in the United Kingdom, offering a proactive multiagency approach for reducing acute hospital admissions and improving the quality of care (NHS England, 2016). The site covered a population of 284,000 people, of which $3 \%$ of the population contribute to $50 \%$ of the cost of healthcare, due to longterm conditions and frequent hospital admissions. At the time of the study, the workforce had recently been organised into five locality-based multidisciplinary teams (MDTs).

The context of the research was subjected to a large number of changes driven nationally, locally and by the research process itself. These included a national workforce planning, and self-assessment tool was launched; the five locality-based teams were physically co-located, which as a change to existing ways care was organised, and the local population data were stratified to enable targeting of complex patients. Structured MDT meetings and care planning processes were implemented and care co-ordination were piloted; a daily team "huddle" meeting and an integrated performance dashboard for the locality system was introduced. The vanguard also implemented a whole system partnership board, which included representatives from all care providers, and a national clinical pharmacist pilot was set up locally. Finally, an organisational development (OD) strategy was developed, and a system-wide workforce development and planning group were set up. 
The research questions addressed were as follows:

(1) What are the skills, knowledge and behaviours that staff working in integrated care need to deliver high quality effective care for patients?

(2) How can current workforce development and planning approaches be improved and delivered to ensure the workforce has capacity and capability to deliver integrated care?

\section{Theory and methods}

The underpinning principle of the research was pragmatism, and so action research was carried out as this approach aligns well with service development where the underpinning delivery model is itself still developing. Action research uses a participatory approach and particularly useful for situations or problems that are unlikely to have a simple, single answer solution, often referred to as "wicked problems". It is usually done in organisations, in this case across organisations, to improve service/outcomes. This approach includes phases of action and evaluation, with findings fed back into the service developmental process and an emphasis on behavioural change (Lewin, 1946; Kemmis et al., 2013; Hart and Bond, 1995). As the models of care were still developing, new local or national initiatives and related findings were incorporated into the local model and research process, along with documentary analysis, such as current workforce plans.

There were three phases of action and evaluation, with the findings from each phase fed back to participants to validate:

Phase 1: literature review; baseline assessment (workforce data collection); initial scoping interviews with system leaders to inform interviews in phase 2 and themes from phase 1 fed into phase 2 in relation with workforce skills, development and planning.

Phase 2: further documentary analysis; interviews and focus groups with managers and workforce; data analysis; themes from phase 2 fed into phase 3 actions; integrated staff training needs analysis was developed and OD strategy was developed.

Phase 3: Interviews with patients receiving care and carers and analysis

Approval to proceed was gained from the University Ethics Committee of the first author, the Health Service Research Authority (HRA) and Research Ethics Committee (REC).

\section{Sampling and recruitment}

Staff participants $(n=30)$ were selected purposively to include system leaders (senior commissioning and operational managers) and frontline workers in integrated teams (GPs, community nurses, mental health nurses and social workers). Most participants had many years' experience in health and/or social care and many had long careers in the area with only two who had worked outside of the area prior to their current role. One-third of the participants were female. Patient participants $(n=4)$ had chronic respiratory conditions. Carer participants $(n=4)$ were caring for patients with respiratory conditions $(n=2)$ or mental health conditions $(n=2)$.

System leaders $(n=12)$ were recruited by direct approach; frontline staff were recruited through open invitation via their line managers. Patients and carers were identified by team leaders from localities. A criterion for inclusion was that they had personal or carer experience of services provided by their local integrated team.

Semi-structured interviews and focus groups were held in a setting convenient to participants' workplaces, patients' homes or the university. Interviews and focus groups were 
JICA 30,5

conducted until data saturation was achieved. Interviews were digitally recorded and transcribed verbatim for analysis.

\section{Data collection and analysis}

Secondary data which were reviewed as part of the research process included national workforce data, local data from all providers were available (including GP practices), current workforce models, performance and outcomes reports and job descriptions. This and themes derived from system leader meetings on workforce informed the development of the questions used in the semi-structured interviews and focus groups.

In total, 17 semi-structured interviews were conducted. Of these, 12 were one-to-one and five with 2-4 respondents with service users and carer(s) or professionals with interrelated roles. of the individual interviews, three took place over the telephone; all others were face-to-face. Face-to-face interviews were held in a variety of venues, including the university, respondents' workplace setting and (in the case of service user and carers) people's homes.

In total, four focus groups were also conducted. These took place with 4 of the 5 new community integrated teams with 4-8 participants in each group, including GPs, social care staff, consultant geriatricians, managers, nurses, social workers, pharmacists, community support workers, practice managers, consultant, carers and service users, MDT co-ordinators and voluntary sector staff. The informed, written consent was obtained in accordance with the ethical approval obtained for the study.

Qualitative data underwent thematic analysis to identify recurring themes, employing constant comparison (Dye et al., 2000) to the point of saturation. A member of the research team, who was not directly involved in conducting interviews or facilitating groups, analysed the data manually, coding themes using an iterative and approach to thematic analysis. Emerging themes were discussed with the Principal Investigator and research team as part of the validation process.

\section{Results \\ Theme 1: Achieving teamwork and integration}

Frontline respondents were positively engaged with the developing model of care and identified several skills, behaviours and opportunities. They felt that a better overall "offer" had been put in place for service users with complex needs, and that the workforce's existing skills and knowledge were better utilised, with a more efficient response to patients and more effective communication between professionals:

There's been some things that would have taken weeks, really, to organise. . . Now we kind of do it in ten minutes in the office. (Pharmacist)

In the past. . you would have gone out, done your assessment, and then have had to make many phone calls to get to speak to the person you needed to ... [but] now you're in the office, you speak to. . . the managers. It's allocated. You have a conversation with who it's allocated to and you go out and everything is done together in front of the patient. (Community nurse)

Co-location of care providers from different organisations and professions during the course of the vanguard developments, whilst initially challenging, was reported to provide a more satisfying working experience. This included enhancement of knowledge, skills and behaviour, improved mutual understanding of roles and expertise, better links between primary and community-based social and healthcare sectors and a proliferation of opportunities for formal and informal co-working, ranging from joint home visits to impromptu case discussion. 
The benefit for me ... is about having that constant contact. I mean, you can share an office with somebody and you have that informal discussion, don't you? . . It's about, "Well, I'm struggling with this-what do you think?" Or "This person has this [issue]; I'm not quite sure because that's not my area of expertise. (Community nurse)

Examples of views of others' ideas and learning were transformed by their exposure to closer working between social care and healthcare workers:

For me, as a social worker ... we go out and we see people, but we're not actually physically hands on. . . One of my first joint ventures was [with] a woman who was in palliative [care]. . . She wanted to stay at home, and we made it so that she could stay at home. . . And I thought, "Eh? They're actually giving injections!" (Social worker)

Many respondents felt that their working life had changed significantly in relation to their notion of teamwork, asking "what team do now I belong to?"; "who is or isn't part of this team?" The concept of "teamness" emerged spontaneously from the frontline team focus groups. Respondents were explicitly encouraged to identify the specific skills and knowledge they felt they needed in relation to teamwork and any training, but frontline staff did not consistently articulate this as any specific training needs or skill requirements in relation to the new model of care or teamworking.

Those in more senior positions, meanwhile, tended to talk about leadership styles and behaviours of middle managers and frontline staff who were responsible for "their" resources for "their" organisations:

If you're the kind of manager that trusts your staff, and you know that [they] are more effective spending time out in the community with patients, rather than at their desk, and you trust them to do their job, and you might only see them once a week for supervision. (Senior manager).

Professional "silos" were seen as obstacles to smoother integration and teamwork, with staff in new roles, feeling "isolated":

I can feel quite isolated, ... I'm often out of the office and out in GP practices, and my team is everywhere else. So sometimes ... I sit in my little corner, and because there's like loads of people around ... Even when I'm in my big room, I can feel isolated. (Clinical Pharmacist)

Structured MDT meetings for patients who had complex needs were implemented with coordinated multi-professional care plans. Considerable variance was reported in terms of the skills perceived to be needed to operate within an MDT setting and to work collaboratively on care plans. Respondents also found it difficult to agree a common definition of "care coordination" or whose role it was. Differing professional backgrounds had inherent differing definitions, such as whether this was an ongoing role or whether it was more of a care navigator who once the care was agreed, could withdraw. Several respondents felt that care co-ordination was a skill rather than a role, whereas others thought care co-ordination was the model of care.

Several respondents commented on the logistical challenges, which hampered their ability to work in an integrated way. The most commonly mentioned concerned IT systems and the lack of integration between patient records and other systems. Respondents understood data governance challenges but repeatedly referred to slow progress towards establishing protocols for data sharing. Many, however, had through co-location and the emergent team culture, developed much stronger face-to-face communication about patients:

The social worker who sits opposite me is going to see a patient, she'll say, "Oh, are they in hospital?" And I can just check on the hospital system - yeah, they're on [Ward Name] or whatever.... Apparently, I'm not allowed to give that information out, because of the governance issues around the fact that I have use of the hospital computer, and I shouldn't be telling social workers that. ... (District nurse)
Workforce skills

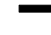


JICA 30,5

98

In total, three patients with complex physical needs and four carers were interviewed. Respondents were generally satisfied with the services delivered, saying they were wellcoordinated particularly in relation to complex physical needs. For example, a patient with diagnoses of chronic pulmonary disease (COPD), diabetes, cardiovascular disease and cancer reflected on coordination of his/her care and co-location of some teams:

They all work in the same office, so they more or less see each other (Patient).

Other parts of the workforce were seen as less effective at care coordination. One couple (patient/carer) complained of receiving unnecessary appointments from the GP practice when

I've told them-I'm under the hospital. (Patient)

A training needs analysis based on a validated tool (Hicks and Hennessy, 2011) was developed for phase 3, but analysis of the interview and focus group data suggested that frontline staff were not yet clear about what their training needs due to lack of role clarity. An OD process was therefore developed, involving Myers Briggs personality assessment (The Myers and Briggs Foundation, 2020), one-to-one coaching for frontline leaders and middle managers and team coaching. OD leads from each organisation were identified to facilitate this plan. Subsequent data suggested a profound "culture shift" within the workforce, with evidence of transformational learning, particularly in senior frontline staff, who through the process of change had begun to both think about and perform their role, in radically different ways beyond prescribed "top-down" pathways. Some respondents subsequently spoke about teams being further ahead in their thinking than their managers:

Where the practitioners are delivering the care, we're challenging their managers to say, "You're not giving us a system that allows us to integrate in the way in which we want. Please do something about it" (Social worker)

Many participants reflected on new workforce cultures developing in health and social care and a pervasive theme of "making a difference" and "no going back" to previous ways of working at the end of the vanguard programme.

\section{Theme 2: emergent demands on capacity and capability}

There was an overwhelming perception, especially among frontline healthcare staff, that additional work had been created for the workforce as a result of regular MDT meetings and the new care-planning processes. Whilst many staff reported they had developed additional skills and could identify unmet need, there were challenges in their capacity to meet unmet needs without additional resources:

I think that together with some of the drivers in government about pushing work back out into the community from the Acute [Hospital] Trusts, the expectation is now that within the community we have that expert resource to facilitate all of that additional work. I would say we absolutely do have the expertise in the community to do that, but if the work continues to increase, that resource is still finite. (Community nurse)

Informal carers were widely recognised by healthcare staff as a crucial resource and their greater integration with professional services as essential to the future of health and social care. This was reflected in the data, and whilst there was substantial support for carers through the Carers Centre, capacity to support for them and the person they care for was a key challenge:

Age UK have a befriender [and] all she does is go out and does befriending. And she is packed [very busy]. Because a lot of it is loneliness. They're on their own - which affects their physical and mental health. . . so they've then employed somebody, which is great. (Carers' centre worker) 
The challenge of capacity in general practice where there was a recognised shortage of GPs was raised by some respondents. They felt that practices were treating MDTs and care plans as "tick box" exercises, with a resistance to unearthing new demand:

You can look at a patient and say, yeah, Mrs X-she's fine. Next one-Mrs Y, yeah, she's fine. You haven't really dug deep and found what the problems are... If it's somebody in their own home, it takes a lot of time. So, yes, one patient might take me six hours, which means I willn't do the ten I should have done, I'll only do two. . .So, that means that eight out of the ten I should have done, have had a more brief care plan. (GP)

\section{Theme 3: delivering holistic and user-centred care}

"Holistic" describes the full range of service user and carers' needs including mental health and emotional well-being. There was considerable variation in how respondents involved in the care of patients with complex physical needs interpreted "mental health", and this affected the importance that they attached to it. The interviewers were clear that mental health issues existed along a spectrum, from low-level anxiety and depression to difficulties requiring more specialised input. Many respondents, who were from a physical health background, suggested that emotional or mental health issues were "rare" or "incidental" to care and something that, if they did arise, was best left to specialists:

We don't get very many referrals around mental health. Quite often it's an incidental finding. (Recovery at Home team member)

We don't get involved with patients with mental health needs ... I mean, obviously, they'll come in for their annual bloods and ECGs and things. But we tend not to see them. It's more the GPs that are managing those. (Practice Nurse)

There were concerns from mental health providers, however, about the perceived mismatch between the primary and community care teams' evaluation of service users' needs and that of the mental health specialists, resulting in a mismatch of expectations of capacity:

I think the issues we've certainly got in mental health older adults [have been] since we raised the profile of dementia. So, the number of referrals coming in has just rocketed [upwards]. And we don't necessarily have the resource to manage that. We're working with agency nurses, which we've never done before in the community. (Community Psychiatric Nurse)

In a joint interview with two long-term, full-time carers of individuals with serious and enduring mental health needs, respondents offered detailed insights into the tensions between supporting the person they cared for and requiring support themselves (from the Carers' Centre). They felt that sometimes their concerns had not sufficiently been addressed by statutory services, and that the emotional and mental strain of caring they had on their own well-being at times had been exacerbated by encounters with both primary and secondary services. Professionals meanwhile reported that the contribution of carers only came very late to their attention, often as part of the new MDT and care planning process:

Sometimes the first time we meet them and that's it - aha! They've never been asked, never been listened to before. . (Community Nurse).

Despite these indications of the extent of mental health issues for patients and carers, it was reported that many respondents had had no or very little training and were not aware of any others in the team who had training. Although on an informal basis they were well used to engaging with patients whose presentation had a mental health component, several respondents suggested it would be helpful if they were able to access training that better equipped them to understand and engage with service users: 
JICA

30,5

I personally think that anyone on the frontline of, you know, service, should have some level of understanding in relation to the things that can affect people's lives. So, if we're talking about simple, low-level training that would benefit an entire integrated team, I can't see how that would be a bad idea... (Social worker)

In total, two carers for people with mental health needs indicated their need for better "education" about their loved one's condition, as well as support, so that they could be more of a "partner in care" with professionals:

We definitely need education. [. . .] that I think is important. Maybe when we start with that, and then they will realise what we're actually doing. (Carer)

Recent policy initiatives around co-production (NHS Leadership Academy, 2019; Seale, 2016) go beyond involving service users and carers in formulating care plans, inviting them to meetings or assisting them to make choices around services. The understanding and skills for co-production and shared decision-making varied, and some respondents queried whether most patients really "want" to be more involved in actively planning their care. There was a common perception that it was either inappropriate for patients and carers to participate in MDT meetings, for practical reasons if not also in principle, or that patients were in fact unwilling to participate:

I think we only bring them in when there's a need. We don't bring them in at every point because we'd have six, seven people-eight people-to talk about how we can develop a care plan with the patient in front of you, and it's difficult to do that. (Multidisciplinary team co-ordinator)

The big problem is the patients not wanting to engage. (Community Nurse)

Paradoxically, MDT meetings were also perceived as promoting a shift away from the coproduction that previously took place more informally with the patient:

Some of the issues that I would just address in a patient's house, immediately, if there was something wrong - sometimes.... I'm having to, like, pause and think, well, right, hold on. I'll wait until Wednesday and discuss it at the MDT. So, I'll say to the patient, Look, oh... We've got a big meeting on Wednesday, the doctor is going to be there, the social worker will be there. Is it okay if I discuss your case? (Senior Community Nurse)

In relation to the skills and training implications of working in a more co-productive way, respondents reported that the way they had been trained previously meant they were not used to or were fearful of working a more co-productive fashion:

[In] general nursing, you've trained to kind of go and do things to people. And we're really good at that. But we're not very good at going and showing people to do things themselves. Or if we are good at it, I think a lot of the time people don't feel empowered and [are] frightened. (Senior Community Nurse)

The service users and carers validated this finding by reporting their expectations of professionals and care plans as being largely prescriptive:

Interviewer: And she helps you make the decisions and that sort of thing?

Patient: Yes. And [she] tells me if I'm doing the right thing or the wrong thing.

\section{Discussion}

In summary, three overarching themes were identified from the final data analysis in relation to the skills, knowledge and behaviours required:

(1) Achieving teamwork and integration, 
(2) Managing demands on capacity and capability and

(3) Delivering holistic and user-centred care.

Sub-themes included faster and more joined-up working, IT systems and skills, leadership, professionals "learning from each other", generalism vs specialism, care co-ordination skills, mental health and well-being skills, co-production, meeting unmet needs and the role of carers.

Due to the size of the defined "teams", some participants did not really understand who was in their team, but, as noted above, the concept of "teamness" was raised by respondents as a simpler way of this thinking about teamwork rather than who was actually in "their team". Other authors have also discussed the idea of "teamness" as a way of conceptualising working in very large teams (West and Lyubovnikova, 2013; Tilden et al., 2016; Shoemaker et al., 2016). In this study, as the OD process embedded, "teamness" and the skills required to work as a team were developing but were at an embryonic stage.

The leadership challenge was that managers were not always adhering to the principles of integration and needed to "see the whites of the eyes" (Senior manager) of their own staff rather than trusting them to work collaboratively with other care providers. Respondents were positive about the new ways of working and the skills they had enhanced and developed as part of the change process, and there was a feeling of "no going back" articulated by many. They valued the opportunity to better mobilise existing specialist knowledge (their own and others in their "team") as well as their generalist skills and to develop new forms of mutual learning and collaboration. Evidence of transformational learning from each other reflects established educational theories around both "deep" and team-based learning as enhancing teamworking (Marton and Saljo, 2005; Michaelson and Sweet, 2012). IT systems and skills were seen to be key for integrated working. Whilst IT training was not reported as a training need, training to support the wider use of telehealth and telecare systems was highlighted by several respondents.

Respondents reported that the new MDT process and care planning had exposed unmet need and was making unforeseen new demands on the workforce, many of which had resource implications. This unintended consequence of the new model illustrates the complexities of enhancing workforce capability, which in turn leads to a greater workforce capacity requirement. Other studies have focussed on how best to ensure a flexible workforce, which is adaptable to changing demands (Nancarrow, 2015), although further work is needed to understand the views of health system performance and provision from the patient perspective.

The final theme included the challenges and opportunities the workforce faces in terms of the ways integrated care is developing. Many respondents recognised the importance of mental health and emotional well-being of service users and carers, both from a policy and professional perspective; however, they also reported a deficit in their knowledge and skills in this area, which aligns with others' findings (Naylor, 2017; Addicott et al., ). Respondents reported poor links between community-based physical care and specialist mental health services as well as a lack of understanding of each other's capacity to deliver.

In the case of co-production, commitment to and/or understanding of the underlying principle varied. Many frontline staff regarded the routine exclusion of patients and/or carers from MDTs, for example, as unproblematic. Recent policy initiatives (Coalition for Collaborative Care, 2016) aim to go beyond involving service users and carers in formulating care plans, such as through inviting them to meetings or assisting them to make choices around services, or even a radical shift about who is "in charge of" health (Boyle and Harris, 2009; Batalden et al., 2016). This latter requires users to be experts and capable of making decisions, with professionals moving from being "fixers to facilitators" (Realpe and 
JICA

30,5

102

Wallace, 2010). If co-production is a genuine goal of new care models, there are considerable training, leadership and support implications. At the time of the research, the national introduction of patient activation measures (PAMs) (NHS England, 2019), which help identify those patients who are ready to engage with developing plans to stay healthy, provides an opportunity for professionals to have a more co-productive relationship with patients.

Whilst the development of new roles is often assumed in many new models (Gilburt, 2016; Crisp and Chen, 2014), the evidence here and elsewhere suggests that valuing and reinforcing existing professional identities can also help to develop trust and recognition and facilitate closer teamworking across professional or organisational boundaries (Mitchell et al., 2011; Rebecca and Brendan, 2015; Liberati et al., 2016). Building effective relationships and establishing a shared commitment for developing care around an individual's needs can support this process. This was echoed in the research to some degree, with several participants reflecting their raised awareness of the differences in their respective roles, and with the challenging discussions on defining the role of the care co-ordinator. Care coordination and what it means to different professional groups, whether it was a skill, a role or a model of care, has also been raised as a challenge in other studies and reflects our findings (Parker and Fuller, 2016; Schultz et al., 2013). In terms of the national policy agenda, further discussion between the professions and care providers may move this forward.

Informal carers are widely and rightly recognised as a crucial resource (OECD, 2011; NHS, 2014; Weatherly et al., 2017) and their greater integration with professional services as essential to the future of health and social care. Other studies have focussed largely on the role of carers' experiences (Cree et al., 2015; Aziz et al., 2016). This study explored additional training carers thought they might need. With our small sample of only four carers, there was evidence of their desire for more clinical knowledge about their loved one's condition, so they could have a greater role in their care. Other studies reflect this finding (Latter et al., 2016; Sin et al., 2017).

In summary, there is evidence from the literature and herein about some of the key skills, knowledge and behaviours required for integrated care. However, how to take forward the workforce planning aspects of integrated care is less clear (Addicott et al., 2015). Workforce data are not currently available across the whole health and social care system, compounded by a lack of workforce skill analysis at local and national levels. One of the particular limitations of the research was accessing accurate, timely workforce data due to issues of "ownership" alongside access to and sharing of the data. This meant that an accurate analysis of workforce capacity could not be made. In addition, individual organisations have their own appraisal and training needs analysis systems, the data which were not shared across the system. After the first cycle of the action research process, an agreement was reached across all providers to carry out a single system-wide training needs analysis; however, due to organisational immaturity and development challenges, the vanguard management team felt the need to postpone this until after the research was completed. Instead, the OD process, which was implemented, was felt to have supported the development of frontline workers and leaders to work creatively and more effectively with partners. It is important to evaluate the impact and cost-effectiveness of new roles and the upskilling of the current workforce if these initiatives are to be replicated in other settings (Lopes et al., 2015; Addicott et al., 2015). Future research should focus on this.

Action research involves continuous and progressive partnership between researchers and the wider community of practice. In this case, engagement with the research was an important part of the OD process, whilst the OD process itself supported the collection of rich qualitative data. The inclusion of focus groups alongside interviews enriched the research, as it allowed interaction between staff from different organisations to learn about team work and "teamness" in a protected environment. An action research approach can help to develop the evidence base and support behavioural change in participants (Latter et al., 2016; Sin 
et al., 2017). There are, however, some potential disadvantages with adopting an action research approach. These include the perceptions that action research is not "science", the findings may not be generalisable to other models and a criticism is that there is poor development of theory (Kemmis et al., 2013). To address these perceived disadvantages, findings from the research were fed back to members of the workforce at each phase to validate in terms of accuracy, validity and relevance to their experience. In addition, research team members not involved in the analysis discussed the findings which allowed a degree of detachment from the data analysis and the generation of a wider range of emergent ideas and themes to be explored.

Purposive sampling aimed to identify staff from different backgrounds and grades as well as carers and service users whose circumstances informed the research from different perspectives. The total number of interviews was dictated by the concept of data "saturation"; however, an acknowledged limitation of the research was the relative shortfall in data obtained about service users' and carers' perspectives on workforce skills. It proved challenging to recruit patient and carer respondents for the research due to the nature of their illnesses. In total, three interviews were held with patients (two with carers present) but were considerably shorter than most of the other interviews largely because of individuals' health conditions. Further research in this area would enhance workforce development. By chance, rich data about the mental health of both carers and the person they cared for emerged and have been used to develop carers' support.

This study did not have its scope consideration of the workforce development and planning challenges for nursing and care staff in residential, nursing care homes and domiciliary services. Given the complexity of multiple independent private providers, however, this part of the workforce is integral to the care pathways for many patients, and in line with the current emerging national focus on this sector, these groups require further examination.

Action research drives action, and the learning has been taken forward through a newlyformed regional group. The aim of this system workforce development group is to inform workforce plans, new role development, training needs analysis and upskilling and the OD process.

\section{Conclusion}

The skills needed to deliver integrated care often already exist within the workforce; the challenge is how these skills are developed, shared and distributed as part of an integrated system of care that spans organisational and professional boundaries. A further challenge is to improve how workforce planning for whole systems is prioritised and subsequently implemented. As health and social care transforms and new models of care develop to create patient-centred, high-quality, efficient care, the workforce delivering this care will need to adapt, developing leadership and teamworking skills to work in a more integrated way across professional boundaries and organisational structures. This transformation will require behavioural and cultural change against a backdrop of a rapidly changing policy context. An OD process, which acknowledges the need to develop people delivering the care as well as the new models of care, is vital.

\section{ORCID iDs}

Karen Giles Dhttp://orcid.org/0000-0002-4225-6915

\section{References}

Addicott, R., Maguire, D., Honeyman, M. and Jabbal, J. (2015), Workforce Planning in the NHS, available at: https://www.kingsfund.org.uk/publications/workforce-planning-nhs. 
JICA

30,5

104

Aziz, N.A., Pindus, D.M., Mullis, R., Walter, F.M. and Mant, J. (2016), "Understanding stroke survivors' and informal carers' experiences of and need for primary care and community health services-a systematic review of the qualitative literature: protocol", BMJ Open, Vol. 6 No. 1, e009244.

Batalden, M., Batalden, P., Margolis, P., Seid, M., Armstrong, G., Opipari-Arrigan, L. and Hartung, H. (2016), "Coproduction of healthcare service”, BMJ Quality and Safety, Vol. 25 No. 7, pp. 509-517.

Better Sunderland HOMEPAGE (2019), "All together better Sunderland”, available at: http://www. atbsunderland.org.uk/ (accessed 9 June-September 2019).

Boyle, D. and Harris, M. (2009), The Challenge of Co-production, NESTA, available at: https://www. nesta.org.uk/report/the-challenge-of-co-production/.

Coalition for Collaborative Care (2016), "Coalition for collaborative care A Co-production model", Coalition for Collaborative Care, available at: http://coalitionforcollaborativecare.org.uk/a-coproduction-model/ (accessed 9 December 2019).

Cree, L., Brooks, H.L., Berzins, K., Fraser, C., Lovell, K. and Bee, P. (2015), “Carers' experiences of involvement in care planning: a qualitative exploration of the facilitators and barriers to engagement with mental health services", BMC Psychiatry, Vol. 15, p. 208.

Crisp, N. and Chen, L. (2014), "Global supply of health professionals", New England Journal of Medicine, Vol. 370 No. 10, pp. 950-957.

Das, P., Naylor, C. and Majeed, A. (2016), "Bringing together physical and mental health within primary care: a new Frontier for integrated care”, Journal of the Royal Society of Medicine, Vol. 109 No. 10, pp. 364-366.

Dept. Health (2013), "Delivering high quality, effective, compassionate care: developing the right people with the right skills and the right values", p. 51, available at: https:/assets.publishing. service.gov.uk/government/uploads/system/uploads/attachment_data/file/203332/29257_ 2900971_Delivering_Accessible.pdf.

Dye, J., Schatz, I., Rosenberg, B. and Coleman, S. (2000), Constant Comparison Method: A Kaleidoscope of Data, The Qualitative Report, 4; 1/2, available at: http://www.nova.edu/ssss/QR/QR4-1/ dye.html.

Gilburt, H. (2016), Supporting Integration through New Roles and Working across Boundaries, The King's Fund, available at: https://www.kingsfund.org.uk/publications/supporting-integrationnew-roles-boundaries (accessed 14 June 2019).

Goodwin, N., Dixon, A., Anderson, G. and Wodchis, W. (2014), Providing Integrated Care for Older People with Complex Needs: Lessons from 7 International Case Studies, Kings Fund, London.

Gullery, C. (2015), “Towards person-centred itnegrated care - the Canterbury journey”, Future Hospital J, Vol. 2 No. 2, pp. 111-116.

Hart, E. and Bond, M. (1995), Action Research for health and Social Care: A Guide to Practice, McGrawHill Education, p. 258.

Health Education (2020), "Health education England shape of caring review", available at: https://hee. nhs.uk/sites/default/files/documents/2348-Shape-of-caring-review-FINAL.pdf (accessed 6 June 2018).

Health Education England (HEE) (2019), "The future of primary care report.pdf", available at: https:// hee.nhs.uk/sites/default/files/documents/The Future of Primary Care report.pdf (accessed 9 September 2019).

Hicks, P.C. and Hennessy, D. (2011), "Hennessy-hicks training needs analysis questionnaire and manual”, p. 59, available at: https://www.who.int/workforcealliance/knowledge/ HennessyHicks_trainingneedstool.pdf.

Kemmis, S., McTaggart, R. and Nixon, R. (2013), The Action Research Planner: Doing Critical Participatory Action Research, Springer, Singapore, Heidelberg, New York, Dordrecht, London, p. 206. 
Kings Fund (2020), "Kings Fund making-integrated-care-happen-kingsfund-mar13.pdf", available at: https://www.kingsfund.org.uk/sites/default/files/field/field_publication_file/making-integratedcare-happen-kingsfund-mar13.pdf (accessed 3 March 2020).

Latter, S., Hopkinson, J.B., Richardson, A., Hughes, J.A., Lowson, E. and Edwards, D. (2016), "How can we help family carers manage pain medicines for patients with advanced cancer? A systematic review of intervention studies", BMJ Supportive and Palliative Care, Vol. 6 No. 3, pp. 263-275.

Lewin, K. (1946), “Action research and minority problems", Journal of Social Issues, Vol. 2 No. 4, pp. 34-46, doi: 10.1111/j.1540-4560.1946.tb02295.x.

Liberati, E.G., Gorli, M. and Scaratti, G. (2016), "Invisible walls within multidisciplinary teams: disciplinary boundaries and their effects on integrated care", Social Science and Medicine, Vol. 150, pp. 31-39.

Ling, T., Brereton, L., Conklin, A., Newbould, J. and Roland, M. (2012), "Barriers and facilitators to integrating care: experiences from the English Integrated Care Pilots", International Journal of Integrated Care, Vol. 12 No. 5, available at: http://www.ijic.org/article/10.5334/ijic.982/ (accessed 10 December 2019).

Lopes, M.A., Almeida, Á.S. and Almada-Lobo, B. (2015), "Handling healthcare workforce planning with care: where do we stand?", Human Resources for Health, Vol. 13 No. 1, p. 38.

Marton, F. and Saljo, R. (2005), “Approaches to learning”, in Marton, F., Hounsell, D. and Entwhistle, N. (Eds), The Experience of Learning: Implications for Teaching and Studying in Higher Education, 3rd (Internet) ed., University of Edinburgh, Centre for Teaching Learning and Assessment, Edinburgh.

Michaelson, L. and Sweet, M. (2012), Team-Based Learning in the Social Sciences and Humanities: Group Work that Works to Generate Critical Thinking and Engagement, Stylus Publishing., Sterling, VA.

Mitchell, R.J., Parker, V. and Giles, M. (2011), "When do interprofessional teams succeed? Investigating the moderating roles of team and professional identity in interprofessional effectiveness", Human Relations, Vol. 64 No. 10, pp. 1321-1343.

Nancarrow, S.A. (2015), "Six principles to enhance health workforce flexibility", Human Resources for Health, Vol. 13 No. 1, available at: http://human-resources-health.biomedcentral.com/articles/10. 1186/1478-4491-13-9 (accessed 9 December 2019).

Naylor, C. (2017), "Physical and mental health", in Amelung, V., Stein, V., Goodwin, N., Balicer, R., Nolte, E. and Suter, E. (Eds), Handbook Integrated Care, Springer International Publishing, Cham, pp. 383-398, available at: http://link.springer.com/10.1007/978-3-319-56103-5_23 (accessed 9 December 2019).

NHS (2014), NHS England Commitment to Carers, available at: https://www.england.nhs.uk/wpcontent/uploads/2014/05/commitment-to-carers-may14.pdf (accessed 9 December 2019).

NHS (2018), NHS England» Vanguards, available at: https://www.england.nhs.uk/new-care-models/ vanguards/ 19 Altogether (accessed 6 June 2018).

NHS (2019a), "England five year forward view", available at: https:/www.england.nhs.uk/wp-content/ uploads/2014/10/5yfv-web.pdf (accessed 10 December 2019).

NHS (2019b), "Next steps on the NHS five year forward view", available at: https://www.england.nhs. uk/wp-content/uploads/2017/03/NEXT-STEPS-ON-THE-NHS-FIVE-YEAR-FORWARD-VIEW. pdf (accessed 10 December 2019).

NHS (2019c), "Improvement evidence from NHS improvement on clinical staff shortages A workforce analysis February 2016", available at: https://assets.publishing.service.gov.uk/government/ uploads/system/uploads/attachment_data/file/500288/Clinical_workforce_report.pdf (accessed 9 September 2019).

NHS (2020a), "Improvement developing people-improving care-010216.pdf", available at: https:// improvement.nhs.uk/documents/542/Developing_People-Improving_Care-010216.pdf (accessed 3 March 2020). 
JICA

30,5

NHS (2020b), NHS interim people plan, available at: https://www.longtermplan.nhs.uk/wp-content/ uploads/2019/05/Interim-NHS-People-Plan_June2019.pdf (accessed 11 March 2020).

NHS England (2016), "New care nodels: Vanguards - developing a blueprint for the future of NHS and care services", p. 64.

NHS England (2019), "Patient activation: at the heart of self-care support", available at: https://www. england.nhs.uk/publication/patient-activation-at-the-heart-of-self-care-support/ (accessed 14 June 2019).

NHS Leadership Academy (2019), "Co-production and community development - a primer", available at: https://www.leadershipacademy.nhs.uk/?attachment_id=18171 (accessed 11 March 2020).

Nuffield Trust (2019), "Nuffield trust what-is-integrated-care-report-web-final.pdf", available at: https://www.nuffieldtrust.org.uk/files/2017-01/what-is-integrated-care-report-web-final.pdf (accessed 10 December 2019).

OECD (2011), "Policies to support family carers", Help Wanted?, OECD Publishing, pp. 121-158, available at: http://www.oecd-ilibrary.org/social-issues-migration-health/help-wanted/policiesto-support-family-carers_9789264097759-9-en (accessed 9 December 2019).

Parker, S. and Fuller, J. (2016), "Are nurses well placed as care co-ordinators in primary care and what is needed to develop their role: a rapid review?", Health and Social Care in the Community, Vol. 24 No. 2, pp. 113-122.

Realpe, A. and Wallace, L. (2010), "What is co-production?", available at: http:/personcentredcare. health.org.uk/sites/default/files/resources/what_is_co-production.pdf (accessed 9 Dec 2019).

Rebecca, M. and Brendan, B. (2015), "Professional diversity, identity salience and team innovation: the moderating role of open mindedness norms", Journal of Organizational Behavior, Vol. 36 No. 6, pp. 873-894.

Schrijvers, G. and Goodwin, N. (2010), "Looking back whilst moving forward: observations on the science and application of integrated care over the past 10 years and predictions for what the next 10 years may hold", International Journal of Integrated Care, Vol. 10, available at: https:// www.ncbi.nlm.nih.gov/pmc/articles/PMC2948683/ (accessed 10 December 2019).

Schultz, E.M., Pineda, N., Lonhart, J., Davies, S.M. and McDonald, K.M. (2013), “A systematic review of the care coordination measurement landscape”, BMC Health Services Research, Vol. 13 No. 1, p. 119.

Seale, B. (2016), Patients as Partners. Building Collaborative Relationships Among Professionals, Patients, Carers and Communities, The Kings Fund, available at: https://www.kingsfund.org. uk/publications/patients-partners (accessed 11 March 2020).

Shoemaker, S.J., Parchman, M.L., Fuda, K.K., Schaefer, J., Levin, J., Hunt, M. and Ricciardi, R. (2016), “A review of instruments to measure interprofessional team-based primary care", Journal of Interprofessional Care, Vol. 30 No. 4, pp. 423-432.

Sin, J., Gillard, S., Spain, D., Cornelius, V., Chen, T. and Henderson, C. (2017), "Effectiveness of psychoeducational interventions for family carers of people with psychosis: a systematic review and meta-analysis", Clinical Psychology Review, Vol. 56, pp. 13-24.

The Myers and Briggs Foundation (2020), The Myers and Briggs Foundation, available at: https:// www.myersbriggs.org/home.htm?bhcp $=1$ (accessed 3 March 2020).

Tilden, V.P., Eckstrom, E. and Dieckmann, N.F. (2016), "Development of the assessment for collaborative environments (ACE-15): a tool to measure perceptions of interprofessional 'teamness", Journal of Interprofessional Care, Vol. 30 No. 3, pp. 288-294.

Weatherly, H., Faria, R., van den Berg, B., Weatherly, H., Faria, R. and van den Berg, B. (2017), "Quantifying informal care for economic evaluation in mental health", in Razzouk, D. (Ed.), Mental Health Economics, Springer, Cham, In Springer, Cham, doi: 10.1007/978-3-319-55266-8-17.

West, M.A. and Lyubovnikova, J. (2013), "Illusions of team working in health care", Journal of Health, Organisation and Management, Vol. 27 No. 1, pp. 134-142. 
WHO (2020a), WHO Global Strategy on People-Centred and Integrated Health Services, WHO, available at: http://www.who.int/servicedeliverysafety/areas/people-centred-care/global-strategy/en/ (accessed 6 January 2020).

WHO (2020b), People-Centred and Integrated Health Services: An Overview of the Evidence, WHO, available at: http://www.who.int/servicedeliverysafety/areas/people-centred-care/evidenceoverview/en/ (accessed 6 January 2020).

\section{Further reading}

Adelman, C. (1993), "Kurt lewin and the origins of action research", Educational Action Research, Vol. 1 No. 1, pp. 7-24.

\section{Corresponding author}

Karen Giles can be contacted at: karen.giles@sunderland.ac.uk

For instructions on how to order reprints of this article, please visit our website:

www.emeraldgrouppublishing.com/licensing/reprints.htm

Or contact us for further details: permissions@emeraldinsight.com 